GENERAL SURVEILLANCE

\title{
From surveillance to action: early gains from the National Violent Death Reporting System
}

\author{
R Campbell, M A Weis, L Millet, V Powell, D Hull-Jilly, H Hackman
}

Injury Prevention 2006;12(Suppl II):ii6-ii9. doi: 10.1136/ip.2006.012450

See end of article for authors' affiliations

\section{Correspondence to:}

Dr R Campbell, Injury

Surveillance Program,

Massachusetts Department

of Public Health, 250

Washington Street, 6th

Floor, Boston, MA 02108-

4619, USA; richard.

campbell@state.ma.us

Accepted 3 August 2006

\begin{abstract}
Objectives: Drawing from the experiences of individual state programs that currently participate in the National Violent Death Reporting System (NVDRS), this article reviews some of the practical benefits that may accrue from the introduction of violent death surveillance systems.

Design: As a state-based surveillance system that uses multiple data sources and relies upon multiple stakeholders, the NVDRS program has fostered an array of initiatives within and among individual state programs. State-based initiatives highlighted in this article were selected on the basis of a purposive sampling strategy intended to illustrate key aspects of program development.

Setting: The NVDRS state programs are in Alaska, California, Colorado, Georgia, Kentucky, Maryland, Massachusetts, New Jersey, New Mexico, North Carolina, Oklahoma, Oregon, Rhode Island, South Carolina, Utah, Virginia, and Wisconsin.

Results: The NVDRS has helped to build alliances and collaborative efforts between key stakeholders, facilitated the recognition of violent death as a public health problem through outreach and media attention, acted as a catalyst for new projects, enhanced surveillance of special populations and utility for evaluation, and identified key circumstances that will target interventions in state prevention planning. Conclusions: The NVDRS has implemented data collection efforts and is beginning to produce and analyze findings. In the process of implementing the data collection system and publicizing findings, state NVDRS programs are realizing other gains that strengthen their surveillance efforts. The use of data for prevention purposes will be the ultimate indicator of program success.
\end{abstract}

$\mathrm{V}$ iolent death is a major public health problem of tremendous scope, in the US and around the globe. In 2002, homicide and suicide claimed the lives of nearly 50000 Americans. $^{1}$ Internationally, the World Health Organization (WHO) in 2002 estimated that more than 1.6 million people suffered violent death per year. ${ }^{2}$ WHO reported that half of these deaths were suicides, while a third resulted from homicide. Prevention of these deaths is a public health priority that can greatly benefit from collaboration across disciplines and geographical boundaries.

In the US, the National Violent Death Reporting System (NVDRS) represents an important recent effort to gather information about violent deaths that may then be used to inform a public health approach to violence. ${ }^{3}$ Through this approach, comprehensive data on violent death are counted upon to define the problem, identify risk and protective factors that might be applied to injury reduction, inform the development and evaluation of intervention programs, and guide policy development and planning.

Although the NVDRS is still in its infancy, the program has demonstrated its effectiveness in several critical areas. This article draws from the experiences of individual NVDRS state programs to highlight the potential benefits from violent death surveillance systems. It is worth noting that reference is made to specific state programs in order to illustrate some of the diverse activities set in motion by the NVDRS. Space limitations prohibit a fuller discussion that includes material from the entire complement of state programs. In addition, while state programs have unique experiences or system features, it should also be pointed out that there are important capacities that cut across state programs. An important part of the NVDRS model, for instance, is a requirement that state programs establish multidisciplinary Advisory Committees in order to ensure the provision of technical advice, oversight, and other support from key stakeholders, including providers and consumers of data. These committees serve to institutionalize partnership arrangements and also to ensure that there is a commitment to the project from important data partners at the time of implementation.

Injury surveillance and the development of the NVDRS As reported by the Surgeon General of the United States, suicide was the ninth leading cause of mortality in the country in 1996, claiming the lives of an average of 85 Americans each day. ${ }^{4}$ Homicides were also significant, accounting for over 19000 fatal injuries in 1997.5 In 1999, the Institute of Medicine estimated in Reducing the burden of injury that unintentional injuries and violence accounted for $30 \%$ of all years of productive life lost before the age of 65 , more than the combined effects of cancer, stroke, or heart disease. ${ }^{6}$ The report noted that dramatic reductions in motor vehicle injuries over 30 years had been achieved through comprehensive understanding of injury risk factors, due in large measure to the Fatality Analysis Reporting System operated by the National Highway Traffic Safety Administration, ${ }^{7}$ and it pointed out a deficit of comparable information for all intentional injuries. It consequently recommended the development of a national fatal intentional injury surveillance system for homicides and suicide.

Taking up the Institute of Medicine challenge, the US Congress, in 2001, approved an appropriation of \$1.5 million to fund the NVDRS as a groundbreaking violent death surveillance initiative. The NVDRS was introduced in 2002 in six state health departments with a goal of providing high quality data applicable to violence prevention of many forms. ${ }^{8}$

Abbreviations: NVDRS, National Violent Death Reporting System; SCIBRS, South Carolina Incident Based Reporting System; SLED, State Law Enforcement Division; WHO, World Health Organization. 
The NVDRS now extends to 17 states: Alaska, California, Colorado, Georgia, Kentucky, Maryland, Massachusetts, New Jersey, New Mexico, North Carolina, Oklahoma, Oregon, Rhode Island, South Carolina, Utah, Virginia, and Wisconsin. It is hoped to ultimately include all 50 states, as well as Washington DC and the US territories, in the NVDRS program.

\section{Early gains from the NVDRS program}

Surveillance systems are customarily seen to be useful if they are able to directly inform intervention programs or aid in assessing the effectiveness of existing intervention programs. ${ }^{9}$ The early experiences of participating state programs suggest that the NVDRS has: (1) helped to build alliances and collaborative efforts between key stakeholders; (2) facilitated the recognition of violent death as a public health problem through outreach and media attention; (3) acted as a catalyst for new projects; (4) enhanced surveillance of special populations and utility for evaluation; and (5) identified key circumstances that will target interventions in state prevention planning. More applications than those identified here may be anticipated as the system matures.

\section{Alliances and key stakeholder collaboration}

The contributions of a range of stakeholders are considered critical to the success of surveillance systems by identifying data sources, generating data, identifying data needs, and helping build public support for a surveillance program. ${ }^{10}$ In the NVDRS program, multiple stakeholders play key roles that bridge the criminal justice and public health systems, integrate data from different sources, and make strategic use of data to guide injury control and prevention.

In Alaska, for instance, the NVDRS project enabled the public health system to implement a state-based database that has the capacity to organize demographic, environmental, and victim-perpetrator information for the analysis of pre-event, event, and post-event data. Although accurate and detailed information was previously available, it was departmentalized and fragmented, making analysis difficult. The Alaska VDRS program has provided a forum around which federal, state, municipal, and community sectors can share information, thereby improving Alaska's ability to analyze violent deaths, develop recommendations for prevention, and undertake concentrated intervention efforts.

The participating organizations and agencies promote a multi-organizational, multi-agency prevention approach to reduce the risk of violence related deaths. Initial collection and abstraction of source records is complicated by law enforcement interagency jurisdictional boundaries. In addition, time delay due to distance and the adjudication processes may have slowed the initial implementation process had alternative strategies not been identified. The Alaska VDRS program used existing relationships developed and sustained by other public health surveillance programs.

In South Carolina, the VDRS program has strengthened and initiated partnerships both within and outside the Division of Injury and Violence Prevention in the South Carolina Department of Health and Environmental Control. Grant partnerships and the creation of the Advisory Council have helped to forge crucial alliances, including collaboration between South Carolina VDRS and the State Law Enforcement Division (SLED) to improve law enforcement reporting to the system. For instance, the South Carolina VDRS program initially planned to receive law enforcement data from the South Carolina Incident Based Reporting System (SCIBRS), part of the Federal Bureau of Investigation's National Incident Based Reporting System. During program implementation, however, it became apparent that VDRS and SCIBRS were not compatible. When the challenge was discussed during an Advisory Council meeting, SLED shared its plans to create a new, web-based, statewide incident reporting system, the South Carolina Information Exchange. By including South Carolina VDRS in the subsequent needs assessment process, almost all NVDRS variables, including narrative and supplementary report information, have become available electronically in South Carolina. The collaboration and sharing of resources of this unprecedented partnership between public health and law enforcement in South Carolina to provide a centralized, electronic source of law enforcement data is a direct result of the alliance created through NVDRS.

In Oregon, the VDRS program has collaborated with partners in the state medical examiner's office and the state Bioterrorism and Preparedness program to identify needs within the medical examiner data system and to work toward the development of a web-based reporting system for medical examiners and deputy medical examiners in the state. The state invested funds from NVDRS to purchase computer hardware, software, training, and programming time. These investments have supported the development of a medical examiner electronic case file that is searchable by manner and cause of death. Eventually, the medical examiner data system will be electronic, with web-based reporting. The current improvements help every public health program in the state, and this work has deepened the public health and medical examiner partnership in Oregon.

\section{Public outreach and dissemination}

Another important function of injury surveillance systems is to communicate findings and bring attention to problems that may otherwise receive little recognition. Dissemination of findings is important for problem recognition, education, and policy influence.

In Virginia, publication of the annual report on violent death findings in October 2005 was accompanied by a press release from the Office of the Commissioner of Health and garnered significant media attention. Following the release of the report, Virginia VDRS staff responded to approximately 20 requests for interviews with newspapers or talk radio, and the report drew prominent coverage from the Washington Post. This attention, in turn, generated calls and inquiries from the public about the project and the report. Media responses (talk radio and newspapers) statewide indicated considerable surprise to learn that there were more suicides than homicides in Virginia, indicating the potential of violent death findings to play an important educational function in relation to violent death.

Publication of the Virginia VDRS report also provided an opportunity for outreach to stakeholders and policy makers. Among the recipients of the report were: national representatives to Congress, state Senators and Delegates, the Governor, the Virginia State Police, directors of state agencies, sheriffs, chiefs of police, directors of local departments of social services, directors of community services boards (agencies providing mental health services), directors of court service units in the department of juvenile justice, victim witness program directors, commonwealth attorneys, domestic violence service providers, child advocates, area councils on aging, directors and nurse managers in local health departments. To date, Virginia VDRS data have been presented to sheriffs, chiefs of police, local medical examiners, and community groups interested in domestic violence prevention.

South Carolina has also successfully disseminated VDRS data to stakeholders and the public. Two statewide violence prevention planning meetings have been convened in conjunction with the release of key publications. In 2004, the Violence Prevention Strategic Planning Meeting introduced the NVDRS to stakeholders and provided the basis for initiating unified violence prevention planning. In 2005, the South Carolina VDRS and its co-sponsors convened 
a statewide violence prevention and data dissemination meeting, attracting approximately 200 participants from a variety of constituencies, including coroners' offices, law enforcement agencies, social services, public health, grassroots programs, and state and local agencies. This and an earlier 2004 conference were driven by data and findings from the South Carolina VDRS. Keynote speakers and workshop were comprised of national, regional, and state experts in their fields. Sessions were planned around suicide prevention, homicide prevention, sexual assault, and domestic violence prevention. The Prevention and Data Dissemination meeting increased the visibility and utilization of the state VRDS program for programmatic efforts and created the environment for diverse stakeholders to forge new partnerships for prevention and child death review-all issues addressed by the NVDRS. The South Carolina VDRS program subsequently received requests for additional analysis and invitations to present to special groups, including community coalitions and county sheriff task forces, and stakeholders also referred media to the VDRS for data, as well. Significantly, the meetings increased interaction among stakeholders who traditionally do not meet, such as coroners and local mental health professionals.

\section{Catalyst for new projects}

The NVDRS program has also demonstrated the ability of surveillance systems to inspire and spark the development of new projects.

In South Carolina, partnerships created through the state VDRS program provided the impetus for developing a system to statistically link health and human services databases with injury and violence data (including the South Carolina VDRS database) in order to identify causal relationships and trends in intentional injury. A grant partner, the Office of Research and Statistics, State Budget and Control Board, holds (through law and memoranda of agreement) multiple databases for various state agencies, thereby facilitating data exchanges. Software tools permit linking data from the various sources and defining multidimensional aggregates of data, termed "cubes", that permit analysis, layering, and drill-down in a user friendly mode through web enabled secure sites. This technology gives planners and policy makers the ability to quickly and easily create tables and analyses that previously required costly analyst support and time. Violence information (including demographics, socioeconomic status, and cause and severity of injury) has been linked to all-payer inpatient hospitalization, emergency room visit, ambulatory surgery, vital records death data, and Department of Mental Health client enrollment files. Injuries and fatalities can be analyzed in relation to a number of variables, such as day of week, season, race, gender, and injury descriptors. Variables also indicate whether individuals had been clients of the Department of Mental Health or been diagnosed with drug or alcohol condition, asthma, epilepsy, diabetes, or heart disease, along with other risk factors for injury. The data linkage provides access to violence analysis that was not previously available.

A separate submodule of the cube has been created for South Carolina's VDRS data. This allows independent analysis of state VDRS data, as well as the ability to include VDRS variables to be included in the total cube if desired. By creating these variables from the VDRS database, they may be connected to other data sources (such as mental health data) to detail the larger picture of the victim's life and past experiences with a mental health facility, or previous law enforcement arrest that could show trends or patterns in behavior. In addition to being the catalyst for cube development, the South Carolina VDRS data is enhanced and expanded through inclusion in the cube.
Enhanced surveillance of special populations and utility for evaluation

In Massachusetts, the VDRS database has, among other important additions, provided the state with the capacity for expanded suicide and homicide surveillance among several unique populations, including foster children and youth and adults in-custody. Massachusetts VDRS data have also improved the state's surveillance of fatal events due to shaken infant syndrome, a diagnosis which currently lacks an International Classification of Diseases, 10th Revision (ICD-10) code and which is difficult to identify in traditional data sets. This expanded capacity, and the resulting data, have been used in several successful grant applications to secure funding for violence prevention activities targeting these populations. Without the NVDRS, interventions aimed at reducing violent events in these select groups would be difficult, if not impossible, to evaluate.

\section{Planning and resource utilization}

In Oregon, older adult suicide was identified as a serious public health problem in 2002. A planning process was launched that year at the same time that the state began participating in the NVDRS. As a result, the state established additional data variables to better define health circumstances associated with older adult suicide cases. Those data were used with other data to develop an epidemiologic profile of older adult suicide in Oregon. Several strategies and activities in Oregon's Older Adult Suicide Prevention Plan are informed by Oregon's VDRS data. The plan calls for targeting primary healthcare providers for training, as well as integration of mental health services into primary care practices. In addition, because the state VDRS data revealed that the majority of older adults dying by suicide were currently married, in relationships, or living with someone, the plan will prioritize educating family members and friends about depression and suicide. The Oregon VDRS program has promoted the plan and provided newspaper, radio, and television interviews to Oregon Public Broadcasting, National Public Radio, and the Oregonian. Following this media work, the Substance Abuse and Mental Health Services Administration granted the state $\$ 100,000$ to use in implementing prevention strategies that target the older adult population.

Oregon is also working to find ways to decrease the need for long term care among older adults. Data from the Oregon VDRS and the Behavioral Risk Factor Surveillance system indicate that among older adults in Oregon, the combination of depression and health problems has a strong association with completed suicide. These comorbid factors can also bring about entry into long term care. The Oregon VDRS staff will participate in a workgroup to use data and research on best practice to identify what practices could be taken to scale in the state.

In South Carolina, in addition to forming the basis for statewide violence prevention strategic planning, data from the South Carolina VDRS has been used in the development of the state's suicide prevention plan. Program staff was invited to join the state suicide prevention coalition in order to provide data guidance to planning and implementation of state programs and initiatives. Staff from the South Carolina VDRS has also been invited to present to county level community organizations in order to provide data and direction at the local level.

\section{DISCUSSION}

The World Health Organization has emphasized that reliable data on violence are needed for planning, monitoring and advocacy, and understanding of the problem. ${ }^{2}$ In the US, the NVDRS has in a short time demonstrated that even at early stages, injury surveillance systems can produce tangible benefits that may extend beyond the anticipated gains that 
motivated the surveillance initiative. Surveillance systems help forge important alliances between multiple stakeholder groups that have strategic value for problem solving, outreach, and planning. They may stimulate new methods of data collection and promote improvements in information systems and databases, enhancing data analysis and the exchange of information. In addition, they provide an important mechanism for raising awareness among a variety of audiences about violence as a public health problem. Surveillance systems may also alert policymakers and the public health community to problems not previously recognized, including injuries in special populations.

However, there are also a number of key challenges that will require attention as the system moves into maturity. One challenge facing individual state programs is finding ways to engage a new set of partners from across disciplines in prevention while maintaining a timely, high quality surveillance system. The partnerships necessary for applying surveillance data to prevention may not always be those that have been involved in the technical aspects of surveillance. These partners, many of which are outside government and have worked for years with little support, may wonder why public health is suddenly interested in violence. Building the necessary relationships for surveillance requires time, particularly since many potential partners have not previously considered the usefulness of data. It is important for the NVDRS that state programs find ways to maintain high quality databases and undertake the initiatives demanded for public health prevention.

A second challenge is sustaining public attention and interest in target communities. The current principal vehicle for public outreach consists of reports on annual findings. Such reporting brings the problem of violence to public attention, but is unlikely to generate ongoing and systematic consideration of the causes of violence and potential forms of intervention. It should be possible to improve in this area as the NVDRS databases are used by outside researchers and stimulate systematic analysis among multiple partners.

Finally, the greatest challenge facing the NVDRS program will be applying the data to the development of specific intervention programs that reduce the incidence of violent death. At this early date, the NVDRS has not inspired the life saving interventions like those in highway safety following the introduction of the Fatal Accident Reporting System-the surveillance system whose accomplishments most directly inspired NVDRS. While use of data to select appropriate practices to reduce the problem of older adult suicide and target interventions has just begun in Oregon, these applications should generate useful information that was not previously available and that is necessary to engage a public health approach to reduce violence. As the NVDRS program moves forward, it will be vital that findings be directed to those partners who can identify problems, devise practices to reduce risk factors and promote protective factors, target high risk populations, evaluate prevention outcomes, promote awareness, inform public policy, and stimulate research. These will be the methods that will take the data into action.

\section{CONCLUSION}

Injury surveillance systems are counted upon to provide the raw material needed to plan meaningful prevention efforts. It is too early to fully evaluate the direct application of the NVDRS system to primary prevention initiatives. However, experiences from participating state programs indicate that even in the implementation phase, the NVDRS is serving as a catalyst for important process developments that are likely to be essential for prevention. Expertise in assessment, community organizing, planning and cross functional collaboration is needed to translate data into action, and these represent everyday activities for public health departments. By drawing on these

\section{Key points}

- Violence, including homicide and suicide, has gained recognition as a public health problem.

- In the US, the National Violent Death Reporting System (NVDRS) was recently introduced by the Centers for Disease Control and Prevention as a state-based violent death surveillance system.

- In implementing NVDRS, individual state programs are forging important alliances, drawing attention to violent death within a public health context, and making other strategic gains that are likely to enhance the possibilities of future success.

- Existing data systems can be useful components of violent death surveillance systems, and the NVDRS approach may have broader application to other countries.

unique public health skills and functions, the NVDRS program is stimulating diverse efforts that should serve as important groundwork for violent injury prevention.

\section{ACKNOWLEDGEMENTS}

We gratefully acknowledge Loreta McKeown and Daksha Gopal, Massachusetts Department of Public Health, Injury Surveillance Program for their assistance in compiling information for this article.

\section{Authors' affiliations}

R Campbell, Massachusetts Department of Public Health, Injury Surveillance Program, Boston, MA, USA

M A Weis, South Carolina Department of Health, Division of Injury and Violence Prevention, Columbia, SC, USA

L Millet, Oregon Department of Human Services, Division of Health

Services, PO, Oregon, USA

V Powell, Virginia Department of Health, Office of the Chief Medical Examiner, Richmond, VA, USA

D Hull-Jilly, Alaska Department of Health and Social Services, Division of Public Health, Anchorage AK, USA

H Hackman, Massachusetts Department of Public Health, Injury

Prevention and Control Program, Boston, MA, USA

This work was supported by the Centers for Disease Control and Prevention (CDC), National Violent Death Reporting System Grant: MA, U17/CCU122394; SC, U17/CCU422396; OR, U17/CCU022393; VA, U17/CCU322391; AK, U17/CCU023102.

The findings and conclusions in this report are those of the authors and do not necessarily represent the views of the Centers for Disease Control and Prevention.

\section{REFERENCES}

1 Centers for Disease Control and Prevention. Web-based Injury Statistics Query and Reporting System (WISQARS). Atlanta, GA: National Center for Injury Prevention and Control (producer). Available at http://www.cdc.gov/ ncipc/wisqars (accessed February 2006).

2 World Health Organization. World report on violence and health: Summary. Geneva, Switzerland: World Health Organization, 2002.

3 Centers for Disease Control and Prevention. Homicide and suicide ratesNational Violent Death Reporting System, six states, 2003. MMWR Morb Mortal Wkly Rep 2005;54:377-80.

4 U. S. Public Health Service. The surgeon general's call to action to prevent suicide. Washington DC: Department of Health and Human Services, 1999.

5 U. S. Department of Health and Human Services. Healthy People 2010. 2nd ed, Washington DC: US Government Printing Office, November, 2000.

6 Bonnie RJ, Fulco CD, Liverman CT (eds). Reducing the burden of injury: advancing prevention and treatment. Washington DC: National Academy Press, 1999.

7 Brown LH, Khanna A, Hunt RC. Rural vs. urban motor vehicle crash death rates: 20 years of FARS data, Prehosp Emerg Care 2000;4:7-13.

8 Paulozzi LJ, Mercy J, Frazier Jr L, et al. CDC's national violent death reporting system: background and methodology. Inj Prev 2004;10:47-52.

9 Horan JM, Mallonee S. Injury surveillance. Epidemiol Rev 2003;25:24-42.

10 Holder Y, Peden M, Krug E, et al, eds. Injury surveillance guidelines. Geneva: Switzerland, World Health Organization, 2001. 